\title{
A Robust Semi-definite Optimization Based Solution To The Robust Order Reduction Problem For Parametric Uncertain Dissipative Linear Systems
}

\author{
Florin Dan Barb
}

Econometric Institute Report EI 2004 - 39

\begin{abstract}
In this paper we address the problem of reducing the order of a linear system affected by uncertainties from the robust dissipative perspective introduced in [4]. Firstly, we show that all major balanced truncation techniques developed and reported in the literature of the last two decades [21], [22], [31], [24], [27], [33], [34] can be treated in a uniform fashion within the framework of dissipative systems. Accordingly, we shall generalize these results to uncertain dissipative systems. The key role is played by balancing two positive definite robust solutions to the uncertain dissipativity LMIs associated with the linear system in question and its dual. Determining the maximal level of uncertainty for which such two solutions exists and computing them efficiently is well known to be NPhard. Our method is based on determining robust tractable approximations of these NP-hard entities by following the novel method known as Matrix-Cube Theory. The proven results are accompanied by a numerical example.
\end{abstract}

\section{Index Terms}

Dissipativity, balanced truncations, Matrix-Cube theory, generalized singular values.

\section{INTRODUCTION AND MOTIVATION}

$\mathbf{R}$ EDUCED order linear models of dynamical systems are obviously preferred over high-order ones since essential engineering tasks like analysis, computer-based simulations and synthesis can be performed faster and cheaper. The problem of obtaining low-order models from high-order ones has been an active research filed over the past two decades. Not surprisingly, there exists a wealth of literature published on this topic and since the inventory of even the most significant published results is beyond the goal of this paper, we kindly invite the "less familiar with the subject" reader to consult a recently published survey [23] and the references therein.

Most order reduction techniques reported so far in the literature have a common starting point - the high-order model is exactly known. The reality is substantially different. For example, when such a nominal is obtained from first-order principles, it is a convenient but imperfect description of the dynamical process under study. It does not account for nonlinear behaviors which are neglected by linearizing more complex nonlinear differential equations around certain equilibrium points, neglected high-frequency behaviors, etc. When such a nominal model is obtained from system identification techniques applied to I/O data collected from physical measurements done on the process in question, the system matrix realization coefficients are also corrupted by uncertainty caused by the very imperfection of the measurement process itself. A

Florin Barb is with Erasmus University, Faculty of Economical Sciences, Department of Mathematics, Burgemeester Oudlaan 50, P. O. Box 1732, Rotterdam 300DR, The Netherlands, barb@ few.eur.nl 
specific feature of the uncertainty affecting identified high-order models has been motivating us to study the order reduction problem from the robust perspective. Specifically, assume that the following set of $\mathrm{I} / \mathrm{O}$ data is known :

$$
\begin{aligned}
U_{N} & =\left[\begin{array}{llll}
u(1) & u(2) & \cdots & u(N)
\end{array}\right] \\
Y_{N}^{\kappa} & =\left[\begin{array}{llll}
y^{\kappa}(1) & y^{\kappa}(2) & \cdots & y^{\kappa}(N)
\end{array}\right], \kappa=1, \cdots, K
\end{aligned}
$$

where $u \in \mathbb{R}^{m}$ is a test input signal of length $N$ and $y^{\kappa} \in \mathbb{R}^{p}$ are the corresponding measured system outputs, assumed that the experiment has been repeated $K$-times under the same conditions. Let now

$$
\mathbf{Y}_{N}=\left[\mathbf{y}_{K}(1), \mathbf{y}_{K}(2), \cdots, \mathbf{y}_{K}(N)\right]
$$

with $\mathbf{y}_{K}(\cdot)=\frac{1}{K} \sum_{k=1}^{K} y^{k}(\cdot)$ denote the mean-value of the output measurements. By assuming the noise Gaussian white with zero mean, the $N$-experimental values will respect a Gaussian-sort distribution centered in the median. Clearly, the bigger are : the length $N$ of each experimental set of values $y^{\kappa}(\cdot)$ and the larger is $K$ the number of available experiments, the closer comes $\mathbf{y}_{K}(\cdot)$ to the true expectation of $y(\cdot)$ - the output function of a certain "nominal system " with coefficients corrupted by Gaussian white noise. Let now such a nominal system obtained from system identification techniques applied to the pair $\left(U_{N}, \mathbf{Y}_{N}\right)$ be described by

$$
\begin{aligned}
\sigma x(t) & =\mathbf{A} x(t)+\mathbf{B} u(t), x(t)=\xi \\
y(t) & =\mathbf{C} x(t)+\mathbf{D} u(t)
\end{aligned}
$$

where $\mathbf{A} \in \mathbb{R}^{n \times n}, \mathbf{B} \in \mathbb{R}^{n \times m}, C \in \mathbb{R}^{p \times n}, \mathbf{D} \in \mathbb{R}^{p \times m}, x(\cdot) \in \mathbb{R}^{n}$ is the state function, $u(\cdot) \in \mathbb{R}^{m}$ is the system input and $y(\cdot) \in \mathbb{R}^{p}$ is the system output. Here the operator $\sigma$ is interpreted as being either the time differentiation operator when continuous time systems are considered or the advanced unit shift in the discrete-time case. The system (3), (4) can also be parameter dependent in the sense that the true values of the system matrix realization coefficients are of the form $\Sigma=\Sigma(\Sigma, p)$, where $p \in \mathbb{R}^{L}$ is a vector of parameters. In this paper we shall consider the affine parameter dependency case.

A convenient way to model the family of all system that can be identified from all possible system outcomes of infinite length is the following :

$$
\mathcal{U}_{\gamma}^{\boldsymbol{\Sigma}}=\left\{\Sigma \in \mathbb{R}^{(p+n) \times(m+n)}, \Sigma=\Sigma+\sum_{\ell=1}^{L} \delta_{\ell} d \Sigma_{\ell}, \max _{\ell=1, \cdots, L}\left|\delta_{\ell}\right| \leq \gamma\right\}
$$

where $\Sigma=\left[\begin{array}{ll}\mathbf{A} & \mathbf{B} \\ \mathbf{C} & \mathbf{D}\end{array}\right] \in \mathbb{R}^{(n+m) \times(n+m)}$ denote the system matrix realization of (3) and (4), $\Delta=$ $\left[\delta_{1}, \cdots, \delta_{L}\right]$ are uncertainties of level $\gamma$ and $d \Sigma_{\ell}, \ell=1, \cdots, L$ are basic perturbation matrices having all coefficients zero except one (different for every $\ell$ ) which equals the unit. Notice that the number of independent perturbations is at most $(p+n) \times(m+n)$. For convenience we shall consider continuous-time models of the form (3) and (4). All results we shall claim and prove in our paper hold also in discretetime with one notable classical exception ; the reduced order system obtained by truncating dissipative balanced realizations is not in balanced form.

Let now $\mathbf{G}(s)=\mathbf{C}(s I-\mathbf{A})^{-1} \mathbf{B}+\mathbf{D}$ denote the transfer function of the system and let $\mathfrak{S}: \mathbb{R}^{p} \times \mathbb{R}^{m} \rightarrow \mathbb{R}$ be a locally integrable functional referred in the the sequel as the system supply

$$
\mathfrak{S}(y, u)=\left[\begin{array}{l}
u \\
y
\end{array}\right]^{\top} \mathbf{P}\left[\begin{array}{l}
u \\
y
\end{array}\right]
$$

where $\mathbf{P} \doteq\left[\begin{array}{cc}\mathbf{Q} & \mathbf{L} \\ \mathbf{L}^{\top} & \mathbf{R}\end{array}\right] \in \mathbb{R}^{(m+p) \times(m+p)}$ is a symmetric real-valued matrix and for the time being no definiteness is assumed on it. 
Definition 1: (Dissipative system) The system $\Sigma$ is called strictly dissipative with respect to the supply $\mathfrak{S}$ (or $(\Sigma, \mathfrak{S})$ is a strictly dissipative pair) if there exists a positive monotonic non-increasing functional $V: \mathbb{R}^{n} \rightarrow \mathbb{R}_{+},\left(V\left(x_{0}\right)=0\right)$, called (normalized) storage such that

$$
V\left(x\left(t_{2}\right)\right)+\int_{t_{1}}^{t_{2}} \mathfrak{S}(u(t), y(t)) d t<V\left(x\left(t_{1}\right)\right)
$$

for all $t_{1} \leq t_{2}$ and all trajectories $(u, x, y)$ satisfying (3) and (4).

In this paper we shall provide a robust semi-definite optimization based solution to the following main problem:

Problem 1:

Find a reduced-order system with state-space dimension $\ell<n$ described by

$$
\begin{aligned}
\dot{z}(t) & =\mathrm{A} z(t)+\mathrm{B} u(t), z(t)=\zeta \\
y(t) & =\mathrm{C} z(t)+\mathrm{D} u(t)
\end{aligned}
$$

with $\mathrm{A} \in \mathbb{R}^{\ell \times \ell}, \mathrm{B} \in \mathbb{R}^{\ell \times m}, \mathrm{C} \in \mathbb{R}^{p \times}, \mathrm{D} \in \mathbb{R}^{m \times m}$ such that if $\mathrm{G}(s)=\mathrm{C}(s I-\mathrm{A})^{-1} \mathrm{~B}+\mathrm{D}$ is its transfer function then :

1) the reduced order system (8), (9) with system matrix realization $\Sigma=\left[\begin{array}{ll}A & B \\ C & D\end{array}\right] \in \mathbb{R}^{(\ell+p) \times(\ell+m)}$ is strictly dissipative w.r.t. the supply function (6)

2) for such a reduced system $\Sigma$, if $\epsilon>0$, then which is its minimal dimension such that the energy stored in it is the fraction $1-\epsilon$ from the one stored in any system $\Sigma \in \mathcal{U}_{\gamma}^{\Sigma}$,

3) $\forall \Sigma \in \mathcal{U}_{\gamma}$ with transfer function $G(s)$, there exists a global bound $\gamma>0$ measured in the $\mathcal{H}_{\infty}$-norm of the reduction error

$$
\|G(s)-\mathrm{G}(s)\|_{\mathcal{H}_{\infty}} \leq \gamma
$$

4) can we develop computationally tractable algorithms to solve these problems?

Firstly let us state our preference for the reduction method; in this paper we shall use the method of truncating system realizations which are balanced w.r.t to some system I/O invariants. One technical contribution of our paper consists in proving that all major "truncation-of-balanced-realizations" order reduction techniques [21], [22], [31], [24], [27], [33], [34] can be formulated and solved in a unified manner provided by the framework of dissipative linear systems with quadratic supply rates developed in the pioneering works [41], [42]. Having this done, we shall extend this general order reduction method to the class of uncertain systems $\mathcal{U}_{\gamma}^{\Sigma}$. Specifically, our method is based on truncating so-called dissipative balanced realizations, obtained by commonly diagonalizing robust dissipativity certificates of the system. Such certificates are positive definite solutions to the dissipativity LMI and its system theoretic dual and they are robust in the sense that are common for all instances $\Sigma \in \mathcal{U}_{\gamma}$. It is well known that determining these certificates is NP-hard [32]. However, by using new results from the emerging field of robust convex optimization [15], the authors of [4] have shown how one can determine a tight tractable approximation of $\Gamma$ - the maximal level of uncertainty for which such certificates exists together with the certificates in question. The goal of this paper is to show that by applying this method (referred in the sequel as the Matrix-Cube Theory) within the framework of robust dissipative systems one can build a tight suboptimal solution to the Problem 1.

The rest of the paper is organized as follows: this section is followed by one devoted to dissipative systems ; we shall develop the dissipative balanced-truncation method. Various extensions to other reduction results are mentioned and $\mathcal{L}_{\infty}$-norm error bounds are given. The third section is devoted to quickly review the main Matrix-Cube Theory results from [15], while the forth one contains the main robust order reduction result for uncertain dissipative systems that has been logically built until that stage in the paper. The last section contains a numerical experiment and the conclusions are herewith drawn.

\section{Dissipative LinEAR SYSTEM THEORY}

In this section we shall review the basic results on dissipativity for linear systems. 


\section{A. Basic results}

Let $(\boldsymbol{\Sigma}, \mathfrak{S})$ be a dissipative pair, where $\boldsymbol{\Sigma}$ is assumed to be in minimal form and stable, i.e. $(\mathbf{A}, \mathbf{B})$ is controllable, $\left(\mathbf{C}^{\top}, \mathbf{A}^{\top}\right)$ is observable and $\lim _{t \rightarrow \infty} x(t)=0, \forall \xi \in \mathbb{R}^{n}$ ) from (3).

According to [41], [42], every storage function $V(\cdot)$ for $(\Sigma, \mathfrak{S})$ is a quadratic function. This implies in our case that there exist $\mathbf{X} \succ 0$ such that $V(x)=x^{\top} \mathbf{X} x>0$. When the storage function is differentiable w.r.t. the time variable, then (7) holds if and only if the following linear matrix inequality is feasible (i.e. admits a positive definite solution $\mathbf{X}$ )

$$
\left[\begin{array}{cc}
\mathbf{A}^{\top} \mathbf{X}+\mathbf{X A} & \mathbf{X B} \\
\mathbf{B}^{\top} \mathbf{X} & 0
\end{array}\right]+\left[\begin{array}{cc}
0 & I \\
\mathbf{C} & \mathbf{D}
\end{array}\right]^{\top} \mathbf{P}\left[\begin{array}{cc}
0 & I \\
\mathbf{C} & \mathbf{D}
\end{array}\right] \prec 0
$$

The following important result has been proven in various particular versions in [28], [35], [36], [37], [38], [39].

Theorem 1: Let $\mathbf{P} \doteq\left[\begin{array}{cc}\mathbf{Q} & \mathbf{L} \\ \mathbf{L}^{\top} & \mathbf{R}\end{array}\right]$ with $\mathbf{R} \succeq 0$ and let $\widehat{\mathbf{P}}=\left[\begin{array}{cc}\widehat{\mathbf{Q}} & \widehat{\mathbf{L}} \\ \widehat{\mathbf{L}}^{\top} & \widehat{\mathbf{R}}\end{array}\right]$ such that $\widehat{\mathbf{P}}=\mathbf{P}^{-1}$. If $\widehat{\mathbf{Q}} \preceq 0$ then the following statements are equivalent :

1) The pair $(\boldsymbol{\Sigma}, \mathfrak{S})$ is strictly dissipative

2) There exist $\mathbf{X}, \mathbf{Y} \succ 0$ such that

$$
\begin{aligned}
\mathcal{D}_{\boldsymbol{\Sigma}}^{\mathbf{P}}(\mathbf{X})= & {\left[\begin{array}{cc}
I & 0 \\
\mathbf{A} & \mathbf{B} \\
0 & I \\
\mathbf{C} & \mathbf{D}
\end{array}\right]^{\top}\left[\begin{array}{cccc}
0 & \mathbf{X} & 0 & 0 \\
\mathbf{X} & 0 & 0 & 0 \\
0 & 0 & \mathbf{Q} & \mathbf{L} \\
0 & 0 & \mathbf{L}^{\top} & \mathbf{R}
\end{array}\right]\left[\begin{array}{cc}
I & 0 \\
\mathbf{A} & \mathbf{B} \\
0 & I \\
\mathbf{C} & \mathbf{D}
\end{array}\right] \prec 0 } \\
\left.\mathcal{D}_{-\mathbf{\Sigma}^{\top}}^{\mathbf{P}^{-1}} \mathbf{Y}\right)= & {\left[\begin{array}{cc}
-\mathbf{A}^{\top} & -\mathbf{C}^{\top} \\
I & 0 \\
-\mathbf{B}^{\top} & -\mathbf{D}^{\top} \\
0 & I
\end{array}\right]\left[\begin{array}{cccc}
0 & \mathbf{Y} & 0 & 0 \\
\mathbf{Y} & 0 & 0 & 0 \\
0 & 0 & \widehat{\mathbf{Q}} & \widehat{\mathbf{L}} \\
0 & 0 & \widehat{\mathbf{L}}^{\top} & \widehat{\mathbf{R}}
\end{array}\right]\left[\begin{array}{cc}
-\mathbf{A}^{\top} & -\mathbf{C}^{\top} \\
I & 0 \\
-\mathbf{B}^{\top} & -\mathbf{D}^{\top} \\
0 & I
\end{array}\right] \succ 0 } \\
& {\left[\begin{array}{cc}
\mathbf{Y} & I \\
I & \mathbf{X}
\end{array}\right]^{\star} 0 } \\
& {\left[\begin{array}{c}
I \\
\mathbf{G}(\jmath \omega)
\end{array}\right]^{\star} \mathbf{P}\left[\begin{array}{c}
I \\
\mathbf{G}(\jmath \omega)
\end{array}\right] \prec 0, \forall \omega \in \mathbb{R} \cup \pm \infty }
\end{aligned}
$$

This result plays the central role in the dissipative reduction algorithm introduced in our paper, both for nominal as well for uncertain systems.

\section{B. Dissipative balanced realization and dissipative BTs}

1) The physics behind dissipativity: Being given a trajectory $(u, x, y)$ of the system $\Sigma$ and two time instants $t_{0} \leq t_{1}$, we interpret the supply $\int_{t_{0}}^{t_{1}} \mathfrak{S}(y(t), u(t)) d t$ as the work carried on the system in the time interval $\left[t_{0}, t_{1}\right]$ along the trajectory in question, if the supply is positive, and as minus the energy extracted from the system, if the supply is negative.

The standard interpretation of a storage function is that $V(x)$ is the internal energy stored by system in state $x$; with this interpretation, dissipativity means that the work done on the system that is needed to move it from one state to another is at least the resulting change in the internal energy stored by the system, while the excess is thought of to be dissipated by the system.

According to [41], [42], [43], among the associated storage functions there exist the (pointwise) minimal one, called "available storage"

$$
V_{a v}(x)=\sup _{(x, y, u)}\left\{-\int_{0}^{t_{1}} \mathfrak{S}(y(t), u(t)) d t, x(0)=x,\right\}
$$


and the (pointwise) maximal one, called "required supply"

$$
V_{\text {req }}(x)=\inf _{(x, y, u)}\left\{\int_{0}^{t_{1}} \mathfrak{S}(y(t), u(t)) d t, x(0)=0, x\left(t_{1}\right)=x\right\}
$$

Every storage function $V(\cdot)$ for $(\Sigma, \mathfrak{S})$ satisfies satisfies $V_{a v}(x)<V(x)<V_{\text {req }}(x)$ for all $x$. Both the available storage and the required supply have the form $V_{a v}(x)=x^{\top} \mathbf{X}_{a v} x, V_{r e q}(x)=x^{\top} \mathbf{X}_{r e q} x$ where the positive definite matrices $\mathbf{X}_{a v}, \mathbf{X}_{r e q}$ are, respectively, the $\prec$-minimal and the $\succ$-maximal solutions of (10). Accordingly, the set of solutions to (10) is exactly the interval $\left\{\mathbf{X}: 0 \prec \mathbf{X}_{a v} \prec \mathbf{X} \prec \mathbf{X}_{r e q}\right\}$.

Let now $\mathfrak{X}=\left\{\mathbf{X}_{a v} \prec \mathbf{X} \prec \mathbf{X}_{r e q}\right\}$ and $\mathfrak{Y}=\left\{\mathbf{Y}_{r e q} \prec \mathbf{Y} \prec \mathbf{Y}_{a v}\right\}$ be the interval of solutions to (11) and (12). From Lemma 1 [33] we know that

$$
\mathfrak{X}=\left\{\mathbf{X}_{a v} \prec \mathbf{X} \prec \mathbf{X}_{r e q}\right\}=\left\{\mathbf{Y}_{a v}^{-1} \prec \mathbf{Y}^{-1} \prec \mathbf{Y}_{r e q}^{-1}\right\}
$$

Scherer has extended this result in [38] by showing that $\mathbf{X}=\mathbf{Y}^{-1}$ holds $\forall \mathbf{X} \in \mathfrak{X}$ and $\forall \mathbf{Y} \in \mathfrak{Y}$. This fact motivated us to introduce the following concept.

Definition 2: (Dissipative balanced form) Let $\mathbf{X}$ and $\mathbf{Y}$ be two solutions to the LMIs (11) and (12). The system matrix realization $\Sigma$ is called in dissipative balanced form if

$$
\mathbf{X}=\mathbf{Y}^{-1}=\operatorname{Diag}\left(\zeta_{1}, \cdots, \zeta_{n}\right)
$$

The recipe of obtaining balanced realizations of linear systems is known in the literature for roughly three decades. Essentially it amounts to finding an invertible transformation which commonly diagonalizes $\mathbf{X}$ and $\mathrm{Y}$. Since the construction of such a transformation can be found in many papers, we shall not outline it here but tacitly make use of it when needed.

The entities $\zeta_{1}, \cdots, \zeta_{n}$ are positive real numbers, possible with multiplicity. Following the classical line of balanced realization theory we shall call them the dissipative singular values of the system (3),(4). The motivation behind this choice is transparent. Indeed, recall that a storage function represents the measure of the amount of energy that can be stored internally by the system or, alternatively, provides the measure of the amount of energy that can be extracted. In balanced form, the storage function has the following expression

$$
\mathbf{V}(x)=\sum_{i=1}^{n} \zeta_{i} x_{i}^{2}
$$

where $x=\left[x_{1}, x_{2}, \cdots, x_{n}\right]^{\top}$. Accordingly, the positive entity $\zeta_{i}$ "measures" in which manner the $i^{\text {th }}$ component $x_{i}$ of the state-vector $x \in \mathbb{R}^{n}$ participates in the energy storage/extraction process. Clearly, the state-components with high energy storage capacity are characterized by large valued $\zeta$ 's, while the ones providing little contribution to the energy storage have corresponding small $\zeta$ 's.

This natural interpretation of the dissipative singular values points out the idea behind the reduction scheme, namely discard the states with small $\zeta$ 's! Technically this is realized by partitioning $\Sigma$ as follows

$$
\boldsymbol{\Sigma}=\left[\begin{array}{cc|c}
\mathbf{A}_{11} & \mathbf{A}_{12} & \mathbf{B}_{1} \\
\mathbf{A}_{21} & \mathbf{A}_{22} & \mathbf{B}_{2} \\
\hline \mathbf{C}_{1} & \mathbf{C}_{2} & \mathbf{D}
\end{array}\right]
$$

where the partition of $\mathbf{A}, \mathbf{B}$ and $\mathbf{C}$ is induced by according to

$$
\underbrace{\zeta_{1} \geq \zeta_{2} \geq \cdots \geq \zeta_{\ell}}_{\text {large values }} \geq \underbrace{\zeta_{\ell+1} \geq \cdots \geq \zeta_{n}>0}_{\text {small values }}
$$

The subsystem $\boldsymbol{\Sigma}_{1}=\left[\begin{array}{cc}\mathbf{A}_{11} & \mathbf{B}_{1} \\ \mathbf{C}_{1} & 0\end{array}\right]$ represents the energetic dominant part of $\boldsymbol{\Sigma}$. In the corresponding state components $x_{1}, \cdots, x_{k}$ one finds back the amount $\frac{\sum_{i=1}^{k} \zeta_{i} x_{i}^{2}}{\sum_{i=1}^{n} \zeta_{i} x_{i}^{2}}$ from the initial energy stored in the components $x_{1}, \cdots, x_{n}$. This last remark gives us the procedure to find the dimension of the reduced 
order system such that the fraction $\delta$ is lost. Indeed, one has to find the corresponding $\zeta_{1} \geq \cdots \geq \zeta_{n}$ and determine $\ell$ such that

$$
\delta \leq 1-\frac{\sum_{i=1}^{\ell} \zeta_{i} x_{i}^{2}}{\sum_{i=1}^{n} \zeta_{i} x_{i}^{2}}
$$

Then $\frac{\sum_{i=\ell+1}^{n} \zeta_{i} x_{i}^{2}}{\sum_{i=1}^{n} \zeta_{i} x_{i}^{2}}$ represents the energy stored in the state of the discarded subsystem $\boldsymbol{\Sigma}_{2}=\left[\begin{array}{cc}\mathbf{A}_{22} & \mathbf{B}_{2} \\ \mathbf{C}_{2} & \mathbf{D}\end{array}\right]$. The above result represents the answer to the second item of the questions listed in the formulation of Problem 1. We shall extend these results to the case of uncertain systems.

\section{C. $\mathcal{L}_{\infty}$-norm error bounds}

The link with the frequency domain is already established in Theorem 1. The inequality (14) is nothing else than the celebrated Kalman-Yakubovich-Popov "positivity" condition and the left hand side of (14) is nothing less than the Popov function [25]

$$
\Pi_{\Sigma}(s)=\left[\begin{array}{c}
I \\
\mathbf{G}(s)
\end{array}\right]^{\star} \mathbf{P}\left[\begin{array}{c}
I \\
\mathbf{G}(s)
\end{array}\right]
$$

Let now $\widetilde{\mathbf{P}} \doteq\left[\begin{array}{cc}0 & I \\ \mathbf{C} & \mathbf{D}\end{array}\right]^{\top} \mathbf{P}\left[\begin{array}{cc}0 & I \\ \mathbf{C} & \mathbf{D}\end{array}\right]=\left[\begin{array}{cc}\widetilde{\mathbf{Q}} & \widetilde{\mathbf{L}} \\ \widetilde{\mathbf{L}}^{\top} & \widetilde{\mathbf{R}}\end{array}\right] \in \mathbb{R}^{(n+m) \times(n+m)}$. Then the Popov function becomes

$$
\boldsymbol{\Pi}_{\Sigma}(s)=\left[\begin{array}{c}
I \\
(s I-\mathbf{A})^{-1} \mathbf{B}
\end{array}\right]^{\star} \widetilde{\mathbf{P}}\left[\begin{array}{c}
I \\
(s I-\mathbf{A})^{-1} \mathbf{B}
\end{array}\right]
$$

Since A was assume stable, it follows [25] that the Popov function is analytic factorizable i.e. that there exists $\Omega \in \mathcal{R} H_{\infty,+}^{m \times m}, \Omega(s)=I-\mathbf{F}(s I-\mathbf{A})^{-1} \mathbf{B}$ such that

$$
\Pi_{\Sigma}(s)=\Omega^{\star}(s) \widetilde{\mathbf{R}} \boldsymbol{\Omega}(s)
$$

Let $\widetilde{\mathbf{R}}=\widetilde{\mathbf{R}}^{\frac{1}{2}} \widetilde{\mathbf{R}}^{\frac{1}{2}}$. It follows that the Popov function can be written as a product of two spectral factors $\Pi_{\Sigma}(s)=\boldsymbol{\Psi}^{\star}(s) \boldsymbol{\Psi}(s)$, where $\boldsymbol{\Psi}(s)=\widetilde{\mathbf{R}}^{\frac{1}{2}} \boldsymbol{\Omega}(s)$. By making use of the dual LMI (12), one can show that $\mathrm{X}$ and $\mathrm{Y}$ are generalized controllability and observability Grammians of the system with transfer function $\boldsymbol{\Psi}(s)$. Accordingly, the dissipative BT is equivalent with the BT of $\boldsymbol{\Psi}(s)$, where balancing is done by commonly diagonalizing the solution to its controllability and observability Lyapunov matrix inequalities

$$
\begin{aligned}
& \mathbf{A}^{\Psi} \mathbf{X}+\mathbf{X A}^{\top, \Psi}+\mathbf{B}^{\Psi} \mathbf{B}^{\top, \Psi} \prec 0 \\
& \mathbf{A}^{\Psi} \mathbf{Y}+\mathbf{Y A}^{\Psi}+\mathbf{C}^{\top, \Psi} \mathbf{C}^{\Psi} \prec 0
\end{aligned}
$$

where $\Sigma^{\Psi}=\left[\begin{array}{ll}\mathbf{A}^{\Psi} & \mathbf{B}^{\Psi} \\ \mathbf{C}^{\Psi} & \mathbf{D}^{\Psi}\end{array}\right] \in \mathbb{R}^{(n+m) \times(n+m)}$ is the system matrix realization of $\boldsymbol{\Psi}=\widetilde{\mathbf{R}}^{\frac{1}{2}} \boldsymbol{\Omega}$ and $\boldsymbol{\Omega}$ has transfer function $\Omega(s)=I-\mathbf{F}(s I-\mathbf{A})^{-1} \mathbf{B}$.

The main result on determining an upper bound on $\left\|\mathbf{G}(s)-\mathbf{G}_{1}(s)\right\|_{\mathcal{H}_{\infty}}$, where $\mathbf{G}_{1}(s)$ is the transfer function associated with $\Sigma_{1}$ - the energetic dominant part of $\Sigma$ retained by dissipative BT is given by the following

Theorem 2: Being given a stable dissipative system $\Sigma$ with transfer function $\mathbf{G}(s)$ let $\Sigma_{1}$ be its energetic dominant part obtained by dissipative BT. Let $\boldsymbol{\Psi}(s)=\widetilde{\mathbf{R}}^{\frac{1}{2}} \boldsymbol{\Omega}(s)$ be a spectral factor of the Popov function associated with $\Sigma$. Then

$$
\left\|\mathbf{G}(s)-\mathbf{G}_{1}(s)\right\|_{\mathcal{H}_{\infty}} \leq 2 \sum_{j=k+1}^{n} \zeta_{j}
$$

The proof is a simple extension of the main bounded-real (particular case of dissipativity) result from section V in [33] where the main truncation result on generalized Hankel singular values (of the spectral factor in this case) has been applied ( see for instance the basic book [19]) 


\section{BASIC MATRIX-Cube TheOry}

Consider an uncertain LMI with affine box uncertainty

$$
\mathcal{A}^{0}(x)+\sum_{\ell=1}^{L} u_{\ell} \mathcal{A}^{\ell}(x) \succeq 0 \quad \forall\left(u:\|u\|_{\infty} \leq \gamma\right),
$$

where

- $x \in \mathbb{R}^{d}$ is the vector of decision variables;

- $\mathcal{A}^{\ell}(x), \ell=0,1, \ldots, L$, are symmetric $m \times m$ matrices affinely depending on $x$;

- $u_{1}, \ldots, u_{L}$ are perturbations, and $\gamma \geq 0$ is the uncertainty level.

It is known that in general, it is NP-hard to solve (22) or even to check whether a given candidate solution $x$ is feasible. However, (22) admits a computationally tractable conservative approximation which is a system of LMIs in original variables $x$ and additional symmetric matrix variables $X_{1}, \ldots, X_{L}$. Let us write $X \succeq \pm Y$ as a shortcut for the system of two matrix inequalities $X \succeq Y, X \succeq-Y$. The aforementioned conservative approximation of (22) is as follows:

$$
\begin{aligned}
X_{\ell} & \succeq \pm \mathcal{A}^{\ell}(x), \quad \ell=1, \ldots, L ; \\
\gamma \sum_{\ell=1}^{L} X_{\ell} & \preceq \mathcal{A}^{0}(x) .
\end{aligned}
$$

The fact that (23) is indeed a conservative approximation of (22) is evident: if $x$ can be extended by appropriately chosen $X_{1}, \ldots, X_{L}$ to a feasible solution of (23), then from (23a) it follows that $u_{\ell} \mathcal{A}^{\ell}(x) \succeq$ $-\gamma X_{\ell}$ for all $u_{\ell}$ such that $\left|u_{\ell}\right| \leq \gamma$; hence

$$
\mathcal{A}^{0}(x)+\sum_{\ell=1}^{L} u_{\ell} \mathcal{A}^{\ell}(x) \succeq \mathcal{A}^{0}(x)-\gamma \sum_{\ell=1}^{L} X_{\ell} \quad \forall\left(u:\|u\|_{\infty} \leq \gamma\right) ;
$$

the right-hand side matrix in the latter relation is $\succeq 0$ by (23b), so that $x$ indeed satisfies (22).

It turns out that the "level of conservativeness" of the approximation (23) is not too big, provided that the matrices $\mathcal{A}^{1}(x), \ldots, \mathcal{A}^{L}(x)$ are of small ranks.

Proposition 1 (matrix cube theorem [15]): Let $\mu=\max _{x} \max _{\ell \geq 1} \operatorname{Rank}\left(\mathcal{A}^{\ell}(x)\right.$ ). (Note $\ell \geq 1$ in the max!). Then the relation between the feasible sets of (22) and (23) is as follows:

1. If $x$ can be extended to a feasible solution of (23), then $x$ is feasible for (22).

2. If $x$ cannot be extended to a feasible solution of (23), then $x$ is infeasible for (22) with $\gamma$ replaced by $\vartheta(\mu) \gamma$, where $\vartheta(\cdot)$ is certain universal function such that $\vartheta(\mu) \leq \frac{\pi \sqrt{\mu}}{2}$ for all $\mu$ and $\vartheta(1)=$ $1, \quad \vartheta(2)=\frac{\pi}{2}=1.57 \ldots, \quad \vartheta(3)=1.73 \ldots, \quad \vartheta(4)=2$.

In particular, for every set $\mathcal{X} \subset \mathbb{R}^{d}$ one has

$$
1 \leq \frac{\sup \{\gamma:(22) \text { has a solution in } \mathcal{X}\}}{\sup \{\gamma:(23) \text { has a solution in } \mathcal{X}\}} \leq \vartheta(\mu)
$$

provided that the numerator in the fraction is positive.

Remark 1: Sometimes we shall be interested in a sufficient condition for the strict version

$$
\mathcal{A}^{0}(x)+\sum_{\ell=1}^{L} u_{\ell} \mathcal{A}^{\ell}(x) \succ 0 \quad \forall\left(u:\|u\|_{\infty} \leq \gamma\right)
$$

of the semi-infinite LMI (22). Such a sufficient condition can be obtained from (23) by replacing the nonstrict LMI (23b) with its strict version. For the resulting pair of conditions, a statement completely similar to the matrix cube theorem takes place.

A severe practical disadvantage of the tractable scheme presented above is that the sizes of these approximations, although polynomial in the sizes $m, n, p, L$ of the underlying dynamical system and uncertainty 
set, are quite large. However, in [15] has been proven that under favorable circumstances, the sizes of the approximating systems can be reduced dramatically.

System (23a) and (23b) is of the generic form

$$
\begin{aligned}
\mathcal{P}(x) & \succeq 0, \\
U_{\ell} & \succeq \pm \mathcal{Q}_{\ell}(x), \quad \ell=1, \ldots, M, \\
V_{\ell} & \succeq \pm R_{\ell}, \quad \ell=1, \ldots, N, \\
\gamma\left[\sum_{\ell} U_{\ell}+\sum_{\ell} V_{\ell}\right] & \preceq \mathcal{S}(x),
\end{aligned}
$$

where

- $x$ is the collection of the original design variables for (23);

- $U_{\ell}, V_{\ell}$ are additional $K \times K$ matrix variables (for (23), $K=m+n+p, M+N=L$, the $U$-variables are those of $X_{\ell}$ for which $\mathcal{A}^{\ell}[x]$ indeed depends on $x$, while the $V$-variables correspond to those of $X_{\ell}$ for $\mathcal{A}^{\ell}[x]$ in fact does not depend on $Z$ );

- $\mathcal{P}(x), \mathcal{Q}_{\ell}(x), \mathcal{S}(x)$ are affine functions of $x$ taking values in the spaces of symmetric matrices of appropriate sizes, and $R_{\ell}$ are given $K \times K$ symmetric matrices.

Note that in the situations we are interested in, the ranks of the matrices $\mathcal{Q}_{\ell}(x), R_{\ell}$ are small, provided that the ranks of basic perturbation matrices $d A_{\ell}, d B_{\ell}, d C_{\ell}, d D_{\ell}$ are small (as indeed is the case in applications). The undesirable large sizes of the approximating system (24) come exactly from the necessity to introduce large-size "matrix bounds" $U_{\ell}, V_{\ell}$ on the small rank matrices $\mathcal{Q}_{\ell}(x), R_{\ell}$.

Note that in our applications all we are interested in are the $x$-components of the feasible solutions of (24). Thus, for our purposes (24) can be replaced with any x-equivalent system of LMIs-a system of LMIs $\mathcal{L}(x, y) \succeq 0$ in the original variables $x$ and additional variables $y$ such that the set of $x$-components of feasible solutions to the latter system is exactly the same as the set of $x$-components of feasible solutions of (24). What we intend to do is to demonstrate that under favorable circumstances we can build a system of LMIs which is $x$-equivalent to (24), while being "much smaller" than the latter system. The key to our construction is given by the following two observations.

Lemma 1 (see [15, Lemma 3.1 and Proposition 2.1]):

(i) Let $a, b$ be two nonzero vectors. A symmetric matrix $X$ satisfies the relation $X \succeq \pm\left[a b^{\top}+b a^{\top} \mathrm{f}\right.$ and only if there exists positive $\lambda$ such that $X \succeq \lambda a a^{\top}+\frac{1}{\lambda} b b^{\top}$.

(ii) Let $A$ be a $n \times n$ symmetric matrix of rank $k>0$, so that $A=P^{\top} \widehat{A} P$ for appropriately chosen $k \times k$ matrix $\widehat{A}$ and $k \times n$ matrix $P$ of rank $k$. A symmetric matrix $X$ satisfies the relation $X \succeq \pm A$ if and only if there exists $k \times k$ symmetric matrix $\widehat{X}$ such that

$$
\begin{aligned}
& X \succeq P^{\top} \widehat{X} P, \\
& \widehat{X} \succeq \pm \widehat{A} .
\end{aligned}
$$

Now assume that the matrices $\mathcal{Q}_{\ell}(x)$ are of the from

$$
\mathcal{Q}_{\ell}(x)=a_{\ell} b_{\ell}^{\top}(x)+b_{\ell}(x) a_{\ell}^{T},
$$

where $a_{\ell} \neq 0, b_{\ell}(x) \not \equiv 0$ are, respectively, a vector and an affine vector-valued function of $x$. Let also

$$
R_{\ell}=P_{\ell}^{\top} \widehat{R}_{\ell} P_{\ell}: \quad \widehat{R}_{\ell}=\widehat{R}_{\ell}^{\top} \in \mathbb{S}^{k_{\ell}}, \quad k_{\ell}=\operatorname{Rank}\left(R_{\ell}\right)>0 .
$$


Applying Lemma 1, we see that (24) is $x$-equivalent to the following system of constraints in the original variables $x$ and the additional variables $\lambda_{\ell} \geq 0, \widehat{V}_{\ell} \in \mathbb{S}^{k_{\ell}}$ :

$$
\begin{aligned}
\mathcal{P}(x) & \succeq 0, \\
\widehat{V}_{\ell} & \succeq \pm \widehat{R}_{\ell}, \quad \ell=1, \ldots, N, \\
\gamma\left[\sum_{\ell}\left[\lambda_{\ell} a_{\ell} a_{\ell}^{\top}+\frac{1}{\lambda_{\ell}} b_{\ell}(x) b_{\ell}^{\top}(x)\right]+\sum_{\ell} P_{\ell}^{\top} \widehat{V}_{\ell} P_{\ell}\right] & \preceq \mathcal{S}(x)
\end{aligned}
$$

(where $\frac{1}{0} b b^{\top}$ is 0 for $b=0$ and is undefined for $b \neq 0$ ). The resulting system, via the Schur complement lemma, is $x$-equivalent to the system of LMIs

$$
\begin{aligned}
& \mathcal{P}(x) \succeq 0, \\
& {\left[\begin{array}{c|cccc}
X-\sum_{\ell=1}^{M} \lambda_{\ell} a_{\ell} a_{\ell}^{\top} & b_{1}(x) & b_{2}(x) & \ldots & b_{M}(x) \\
\hline b_{1}^{\top}(x) & \lambda_{1} & & & \\
b_{2}^{\top}(x) & & \lambda_{2} & & \\
\vdots & & & \ddots & \\
b_{M}^{\top}(x) & & & & \lambda_{M}
\end{array}\right] \succeq 0,} \\
& \gamma\left[X+\sum_{\ell} P_{\ell}^{\top} \widehat{V}_{\ell} P_{\ell}\right] \preceq \mathcal{S}(x)
\end{aligned}
$$

in the original variables $x$ and additional scalar variables $\left\{\lambda_{\ell}\right\}_{\ell=1}^{M}$ and matrix variables $X,\left\{\widehat{V}_{\ell}\right\}_{\ell=1}^{N}$.

System (27) is $x$-equivalent to our original system (24) and is usually much better suited for numerical processing than the original system. Indeed, as compared to (24), in (27) there are

- a single $K \times K$ matrix variable $X$ and $M$ scalar variables $\left\{\lambda_{\ell}\right\}_{\ell=1}^{M}$ instead of $M K \times K$ matrix variables $U_{\ell}$;

- $k_{\ell} \times k_{\ell}$ matrix variables $\widehat{V}_{\ell}$ instead of $K \times K$ matrix variables $V_{\ell}$, and $k_{\ell} \times k_{\ell}$ LMIs (27c) instead of $K \times K$ LMIs (24c) (recall that $k_{\ell}$ are assumed to be small as compared to $K$ );

- a single LMI (27b) instead of $M$ LMIs (24b). Although the size of LMI (27b) is larger than those of LMIs (24b), the LMI is of very simple arrow structure and is extremely sparse.

\section{The MatriX-Cube Solution to The MAIN PROBlem}

Recall now that our main goal is to obtain robust dissipativity certificates and tight bounds on the maximal uncertainty level for which such certificates exist. The following results is a simple consequence of Theorem 1 .

Theorem 1: Let $\mathbf{P} \doteq\left[\begin{array}{cc}\mathbf{Q} & \mathbf{L} \\ \mathbf{L}^{\top} & \mathbf{R}\end{array}\right]$ with $\mathbf{R} \succeq 0$ and let $\widehat{\mathbf{P}}=\left[\begin{array}{cc}\widehat{\mathbf{Q}} & \widehat{\mathbf{L}} \\ \widehat{\mathbf{L}}^{\top} & \widehat{\mathbf{R}}\end{array}\right]$ such that $\widehat{\mathbf{P}}=\mathbf{P}^{-1}$. If $\widehat{\mathbf{Q}} \preceq 0$ then the following statements are equivalent :

1) The pair $(\Sigma, \mathfrak{S})$ is strictly dissipative, $\forall \Sigma \in \mathcal{U}_{\gamma}^{\Sigma}$, 
2) There exist $X, Y \succ 0$ such that

$$
\begin{aligned}
\mathcal{D}_{\Sigma}^{\mathbf{P}}(\mathbf{X})= & {\left[\begin{array}{cc}
I & 0 \\
A & B \\
0 & I \\
C & D
\end{array}\right]^{\top}\left[\begin{array}{cccc}
0 & \mathbf{X} & 0 & 0 \\
\mathbf{X} & 0 & 0 & 0 \\
0 & 0 & \mathbf{Q} & \mathbf{L} \\
0 & 0 & \mathbf{L}^{\top} & \mathbf{R}
\end{array}\right]\left[\begin{array}{cc}
I & 0 \\
A & B \\
0 & I \\
C & D
\end{array}\right] \prec 0 } \\
\mathcal{D}_{-\Sigma^{\top}}^{\mathbf{P}^{-1}}(\mathbf{Y})= & {\left[\begin{array}{cc}
-A^{\top} & -C^{\top} \\
I & 0 \\
-B^{\top} & -D^{\top} \\
0 & I
\end{array}\right]\left[\begin{array}{cccc}
0 & \mathbf{Y} & 0 & 0 \\
\mathbf{Y} & 0 & 0 & 0 \\
0 & 0 & \widehat{\mathbf{Q}} & \widehat{\mathbf{L}} \\
0 & 0 & \widehat{\mathbf{L}}^{\top} & \widehat{\mathbf{R}}
\end{array}\right]\left[\begin{array}{cc}
-A^{\top} & -C^{\top} \\
I & 0 \\
-B^{\top} & -D^{\top} \\
0 & I
\end{array}\right] \succ 0 } \\
& {\left[\begin{array}{cc}
\mathrm{Y} & I \\
I & \mathbf{X}
\end{array}\right] \succ 0 } \\
& {\left[\begin{array}{c}
I \\
G(\jmath \omega)
\end{array}\right]^{\star} \mathbf{P}\left[\begin{array}{c}
I \\
G(\jmath \omega)
\end{array}\right] \prec 0, \forall \omega \in \mathbb{R} \cup \pm \infty }
\end{aligned}
$$

Notice that the dissipativity condition (28) can be written for arbitrary $\Sigma \in \mathcal{U}_{\gamma}^{\Sigma}$ as follows :

$$
\begin{aligned}
& {\left[\begin{array}{cc}
A^{\top} \mathbf{X}+\mathbf{X} A & \mathbf{X} B \\
B^{\top} \mathbf{X} & 0
\end{array}\right]+\left[\begin{array}{cc}
0 & I \\
C & D
\end{array}\right]^{\top}\left[\begin{array}{cc}
\mathbf{Q} & \mathbf{L} \\
\mathbf{L}^{\top} & \mathbf{R}
\end{array}\right]\left[\begin{array}{cc}
0 & I \\
C & D
\end{array}\right] \prec 0 \Leftrightarrow} \\
& {\left[\begin{array}{ccc}
A^{\top} \mathbf{X}+\mathbf{X} A & \mathbf{X} B+C^{\top} \mathbf{L} & C^{\top} \mathbf{W} \\
B^{\top} \mathbf{X}+\mathbf{L}^{\top} C & \mathbf{L}^{\top} D+D^{\top} \mathbf{L}+\mathbf{Q} & D^{\top} \mathbf{W} \\
\mathbf{W}^{\top} C & \mathbf{W}^{\top} D & -\mathbf{S}
\end{array}\right] \prec 0}
\end{aligned}
$$

where $\mathbf{R} \succeq 0$ has been factorizes as $\mathbf{R}=\mathbf{W S}^{-1} \mathbf{W}^{\top}, \mathbf{S} \succ 0$. Accordingly, let us write down the basic perturbation matrices as $d \Sigma=\left[\begin{array}{ll}d A & d B \\ d C & d D\end{array}\right]$. It follows that (32) has the following form:

$$
\left[\begin{array}{ccc}
(\mathbf{A}+d A)^{\top} \mathbf{X}+\mathbf{X}(\mathbf{A}+d A) & \mathbf{X}(\mathbf{B}+d B)+(\mathbf{C}+d C)^{\top} \mathbf{L} & (\mathbf{C}+d C)^{\top} \mathbf{W} \\
(\mathbf{B}+d B)^{\top} \mathbf{X}+\mathbf{L}^{\top}(\mathbf{C}+d C) & \mathbf{L}^{\top}(\mathbf{D}+d D)+(\mathbf{D}+d D)^{\top} \mathbf{L}+\mathbf{Q} & (\mathbf{D}+d D)^{\top} \mathbf{W} \\
\mathbf{W}^{\top}(\mathbf{C}+d C) & \mathbf{W}^{\top}(\mathbf{D}+d D) & -\mathbf{S}
\end{array}\right] \prec 0(33)
$$

Then the approximate robust counterpart of (33) as given by the Matrix-Cube Theorem is the following

$$
\begin{aligned}
X_{\ell} & \preceq \pm\left[\begin{array}{ccc}
d A_{\ell}^{\top} \mathbf{X}+\mathbf{X} d A_{\ell} & \mathbf{X} d B_{\ell}+d C_{\ell}^{\top} \mathbf{L} & d C_{\ell}^{\top} \mathbf{W} \\
d B_{\ell}^{\top} \mathbf{X}+\mathbf{L}^{\top} d C_{\ell} & \mathbf{L}^{\top} d D_{\ell}+d D_{\ell}^{\top} \mathbf{L}+\mathbf{Q} & d D_{\ell}^{\top} \mathbf{W} \\
\mathbf{W}^{\top} d C_{\ell} & \mathbf{W}^{\top} d D_{\ell} & 0
\end{array}\right], \ell=1, \cdots, L \\
\gamma \sum_{\ell=1}^{L} X_{\ell} & =\left[\begin{array}{ccc}
\mathbf{A}^{\top} \mathbf{X}+\mathbf{X} \mathbf{A} & \mathbf{X} \mathbf{B}+\mathbf{C}^{\top} \mathbf{L} & \mathbf{C}^{\top} \mathbf{W} \\
\mathbf{B}^{\top} \mathbf{X}+\mathbf{L}^{\top} \mathbf{C} & \mathbf{L}^{\top} \mathbf{D}+\mathbf{D}^{\top} \mathbf{L}+\mathbf{Q} & \mathbf{D}^{\top} \mathbf{W} \\
\mathbf{W}^{\top} \mathbf{C} & \mathbf{W}^{\top} \mathbf{D} & -\mathbf{S}
\end{array}\right]
\end{aligned}
$$

in variables $\mathrm{X}, X_{1}, \cdots, X_{L}$.

In a very similar fashion one can write down the approximate robust counterpart for (29) as

$$
\begin{aligned}
Y_{\ell} \succeq \pm\left[\begin{array}{ccc}
d A_{\ell} \mathbf{Y}+\mathbf{Y} d A_{\ell^{\top}} & \mathbf{Y} d C_{\ell}^{\top}+d B_{\ell} \widehat{\mathbf{L}} & d B_{\ell} \widehat{\mathbf{W}} \\
d C_{\ell} \mathbf{Y}+\widehat{\mathbf{L}}^{\top} d B_{\ell^{\top}} & \widehat{\mathbf{L}}^{\top} d D_{\ell}^{\top}+d D_{\ell} \widehat{\mathbf{L}}+\widehat{\mathbf{Q}} & d D_{\ell} \widehat{\mathbf{W}} \\
\widehat{\mathbf{W}}^{\top} d B_{\ell}^{\top} & \widehat{\mathbf{W}}^{\top} d D_{\ell}^{\top} & 0
\end{array}\right], \ell=1, \cdots, L \\
\gamma \sum_{\ell=1}^{L} Y_{\ell} \preceq\left[\begin{array}{ccc}
\mathbf{A} \mathbf{Y}+\mathbf{Y} \mathbf{A}^{\top} & \mathbf{Y} \mathbf{C}^{\top}+\mathbf{B} \widehat{\mathbf{L}} & \mathbf{B} \widehat{\mathbf{W}} \\
\mathbf{C Y}+\widehat{\mathbf{L}}^{\top} \mathbf{B}^{\top} & \widehat{\mathbf{L}}^{\top} \mathbf{D}^{\top}+\mathbf{D} \widehat{\mathbf{L}}+\widehat{\mathbf{Q}} & \mathbf{D} \widehat{\mathbf{W}} \\
\widehat{\mathbf{W}}^{\top} \mathbf{B}^{\top} & \widehat{\mathbf{W}}^{\top} \mathbf{D}^{\top} & -\widehat{\mathbf{S}}
\end{array}\right]
\end{aligned}
$$

in variables $\mathrm{Y}, Y_{1}, \cdots, Y_{L}$ and where $\widehat{\mathbf{Q}} \preceq 0$ has been factorizes as $\widehat{\mathbf{Q}}=\widehat{\mathbf{W}} \widehat{\mathbf{S}}^{-1} \widehat{\mathbf{W}}^{\top}, \widehat{\mathbf{S}} \prec 0$. 
The following result holds:

Theorem 2:

Let $\mathrm{X}, \mathrm{Y} \succ 0$ be the $\mathrm{X}$ - and $\mathrm{Y}$-components of the solution $\left(\mathrm{X}, \mathrm{Y}, X_{1}, \cdots, X_{L}, Y_{1}, \cdots Y_{L}\right)$ to the semidefinite feasibility problem (34), (35) and (36) and (37) and let $\widehat{\Gamma}$ be the maximal level of uncertainty such that (34), (35) and (36) and (37) is solvable. Then

(1 $\mathrm{X}, \mathrm{Y} \succ 0$ are robust dissipative certificates $\forall \Sigma \in \mathcal{U}_{\widehat{\Gamma}}^{\Sigma}$

2 If $\Gamma$ is the true maximal uncertainty level for which (28), (29) have solutions, then $\widehat{\Gamma} \leq \Gamma \leq \frac{\pi}{2} \widehat{\Gamma}$.

Based on Theorem 2 we can formulate an prove the Matrix-Cube theory based result on robust order reduction of uncertain dissipative linear systems.

Theorem 3: Let $\Sigma=\left[\begin{array}{cc}A & B \\ C & D\end{array}\right]$ be an arbitrary instance of the box of system matrix realizations $\mathcal{U}_{\gamma}^{\Sigma}$ and let

$$
\mathcal{P}_{1}: \quad \Gamma=\max _{\gamma>0} \quad \gamma
$$

$$
X, Y \succ 0
$$

$$
\begin{aligned}
& {\left[\begin{array}{cc}
I & 0 \\
A & B \\
0 & I \\
C & D
\end{array}\right]^{\top}\left[\begin{array}{cccc}
0 & X & 0 & 0 \\
X & 0 & 0 & 0 \\
0 & 0 & \mathbf{Q} & \mathbf{L} \\
0 & 0 & \mathbf{L}^{\top} & \mathbf{R}
\end{array}\right]\left[\begin{array}{cc}
I & 0 \\
A & B \\
0 & I \\
C & D
\end{array}\right] \prec 0} \\
& {\left[\begin{array}{cc}
-A^{\top} & -C^{\top} \\
I & 0 \\
-B^{\top} & -D^{\top} \\
0 & I
\end{array}\right]^{\top}\left[\begin{array}{cccc}
0 & Y & 0 & 0 \\
Y & 0 & 0 & 0 \\
0 & 0 & \widehat{\mathbf{Q}} & \widehat{\mathbf{L}} \\
0 & 0 & \widehat{\mathbf{L}}^{\top} & \widehat{\mathbf{R}}
\end{array}\right]\left[\begin{array}{cc}
-A^{\top} & -C^{\top} \\
I & 0 \\
-B^{\top} & -D^{\top} \\
0 & I
\end{array}\right] \succ 0} \\
& {\left[\begin{array}{ll}
Y & I \\
I & X
\end{array}\right] \succ 0}
\end{aligned}
$$

Consider the following semi-definite optimization problem:

$$
\begin{aligned}
& \mathcal{P}_{2}: \quad \widetilde{\Gamma}=\max _{\gamma>0} \gamma \\
& \mathrm{X}, \mathrm{Y} \succ 0 \\
& X_{\ell} \preceq \pm\left[\begin{array}{ccc}
d A_{\ell}^{\top} \mathbf{X}+\mathbf{X} d A_{\ell} & \mathbf{X} d B_{\ell}+d C_{\ell}^{\top} \mathbf{L} & d C_{\ell}^{\top} \mathbf{W} \\
d B_{\ell}^{\top} \mathbf{X}+\mathbf{L}^{\top} d C_{\ell} & \mathbf{L}^{\top} d D_{\ell}+d D_{\ell}^{\top} \mathbf{L}+\mathbf{Q} & d D_{\ell}^{\top} \mathbf{W} \\
\mathbf{W}^{\top} d C_{\ell} & \mathbf{W}^{\top} d D_{\ell} & 0
\end{array}\right] \\
& \gamma \sum_{\ell=1}^{L} X_{\ell} \succeq\left[\begin{array}{ccc}
\mathbf{A}^{\top} \mathbf{X}+\mathbf{X} \mathbf{A} & \mathbf{X B}+\mathbf{C}^{\top} \mathbf{L} & \mathbf{C}^{\top} \mathbf{W} \\
\mathbf{B}^{\top} \mathbf{X}+\mathbf{L}^{\top} \mathbf{C} & \mathbf{L}^{\top} \mathbf{D}+\mathbf{D}^{\top} \mathbf{L}+\mathbf{Q} & \mathbf{D}^{\top} \mathbf{W} \\
\mathbf{W}^{\top} \mathbf{C} & \mathbf{W}^{\top} \mathbf{D} & -\mathbf{S}
\end{array}\right] \\
& Y_{\ell} \quad \succeq \pm\left[\begin{array}{ccc}
d A_{\ell} \mathbf{Y}+\mathbf{Y} d A_{\ell^{\top}} & \mathbf{Y} d C_{\ell}^{\top}+d B_{\ell} \widehat{\mathbf{L}} & d B_{\ell} \widehat{\mathbf{W}} \\
d C_{\ell} \mathbf{Y}+\widehat{\mathbf{L}}^{\top} d B_{\ell^{\top}} & \widehat{\mathbf{L}}^{\top} d D_{\ell}^{\top}+d D_{\ell} \widehat{\mathbf{L}}+\widehat{\mathbf{Q}} & d D_{\ell} \widehat{\mathbf{W}} \\
\widehat{\mathbf{W}}^{\top} d B_{\ell}^{\top} & \widehat{\mathbf{W}}^{\top} d D_{\ell}^{\top} & 0
\end{array}\right] \\
& \gamma \sum_{\ell=1}^{L} Y_{\ell} \preceq\left[\begin{array}{ccc}
\mathbf{A Y}+\mathbf{Y} \mathbf{A}^{\top} & \mathbf{Y} \mathbf{C}^{\top}+\mathbf{B} \widehat{\mathbf{L}} & \mathbf{B} \widehat{\mathbf{W}} \\
\mathbf{C Y}+\widehat{\mathbf{L}}^{\top} \mathbf{B}^{\top} & \widehat{\mathbf{L}}^{\top} \mathbf{D}^{\top}+\mathbf{D} \widehat{\mathbf{L}}+\widehat{\mathbf{Q}} & \mathbf{D} \widehat{\mathbf{W}} \\
\widehat{\mathbf{W}}^{\top} \mathbf{B}^{\top} & \widehat{\mathbf{W}}^{\top} \mathbf{D}^{\top} & -\widehat{\mathbf{S}} \\
\ell=1,2, \cdots, L & &
\end{array}\right]
\end{aligned}
$$

Then

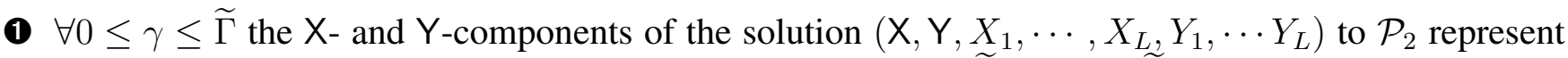
robust solutions to the problem $\mathcal{P}_{1}$ and they are tight up to $\widetilde{\Gamma} \leq \Gamma \leq \frac{\pi}{2} \widetilde{\Gamma}$.

2 Whenever $\gamma>\frac{\pi}{2} \widetilde{\Gamma}$, the semi-infinite system of semi-definite constraints in $\mathcal{P}_{1}$ is unfeasible. 
(3) Let $T \in \mathbb{R}^{n \times n}$ be a balancing transformation which balances $\mathrm{X}$ and $\mathrm{Y}$, i.e.

$$
\mathrm{X}=\mathrm{Y}=\operatorname{Diag}\left(\zeta_{1}, \cdots, \zeta_{\ell}, \zeta_{\ell+1}, \cdots, \zeta_{n}\right)
$$

the diagonal matrix of generalized dissipative singular values (assumed in descending order) and let $\boldsymbol{\Sigma}=\left[\begin{array}{cc|c}\mathbf{A}_{11} & \mathbf{A}_{12} & \mathbf{B}_{1} \\ \mathbf{A}_{21} & \mathbf{A}_{22} & \mathbf{B}_{2 u} \\ \hline \mathbf{C}_{1} & \mathbf{C}_{2} & \mathbf{D}\end{array}\right]$ be the partitioned induced on $\boldsymbol{\Sigma}_{1}$ by the balancing transformation. Let now $\Sigma=\left[\begin{array}{cc}\mathbf{A}_{11} & \mathbf{B}_{1 u} \\ \mathbf{C}_{1 y} & 0\end{array}\right]$. Then $\Sigma$ is the solution to Problem 1 in the sense that $\forall \Sigma \in \mathcal{U}_{\widetilde{\Gamma}}^{\Sigma}$ with transfer function $G(s)$, if $\mathrm{G}$ is the transfer function corresponding to $\Sigma$, then

$$
\|G(s)-\mathrm{G}(s)\|_{\mathcal{H}_{\infty}} \leq 2 \sum_{j=1}^{k} \zeta_{j}+4 \sum_{j=k+1}^{n} \zeta_{j}
$$

Proof: By arriving at this stage in our exposure, the proof is trivial : item $\mathbf{0}$ is clearly a direct application of the mainn Matrix-Cube theory result to (28) and (29). Indeed $\Sigma$ is the energetic dominant counterpart of any system $\Sigma$ in the box $\forall \Sigma \in \mathcal{U}_{\widetilde{\Gamma}}^{\Sigma}$ (see the interpretation of the dissipative singular values which can be extended one-to-one to their generalized counterparts), hence also for $\Sigma$. Accordingly, by truncating $\Sigma$ balanced w.r.t. to the robust dissipative certificates one obtains a robustly reduced order system which satisfies

$$
\begin{aligned}
& \|G(s)-\mathbf{G}(s)+\mathbf{G}(s)-\mathbf{G}(s)\|_{\mathcal{H}_{\infty}} \leq\|G(s)-\mathbf{G}(s)\|_{\mathcal{H}_{\infty}}+\|\mathbf{G}(s)-\mathbf{G}(s)\|_{\mathcal{H}_{\infty}} \leq \\
& \leq 2 \sum_{j=1}^{n} \zeta_{j}+\|\mathbf{G}(s)-\mathrm{G}(s)\|_{\mathcal{H}_{\infty}} \leq 2 \sum_{j=1}^{k} \zeta_{j}+4 \sum_{j=k+1}^{n} \zeta_{j}
\end{aligned}
$$

and claim 3 is also proved.

Let now $\delta>0$. One can find the corresponding $1<\ell<n$ by simply searching for the validity of the condition

$$
\delta \leq 1-\frac{\sum_{i=1}^{\ell} \zeta_{i} x_{i}^{2}}{\sum_{i=1}^{n} \zeta_{i} x_{i}^{2}}
$$

where $x_{i}$ are the states of $\Sigma$ and $x_{i}$ are the states of the arbitrary chosen $\Sigma$, assumed both in generalized dissipative balanced form.

\section{NUMERICAL EXAMPLE}

Let us consider from [29] the following nominal state-space representation of the aero-elastic system including the model of unsteady aerodynamic forces

$$
\begin{aligned}
& \mathbf{A}=10^{3}\left[\begin{array}{rrrr}
0 & 0 & 0.0010 & 0 \\
0 & 0 & 0 & 0.0010 \\
-0.2911 & -0.0012 & -0.0034 & -0.0001 \\
1.8469 & -0.0544 & 0.0202 & -0.0003
\end{array}\right], \mathbf{B}^{\top}=\left[\begin{array}{rrrr}
0 & 0 & -0.1023 & 0.6493 \\
0 & 0 & -0.6493 & 19.5040 \\
0 & 0 & -0.1023 & 0.6493 \\
0 & 0 & 0.6493 & -19.5040 \\
0 & 0 & -8.5104 & 207.1568
\end{array}\right] \\
& \mathbf{C}=\left[\begin{array}{rrrr}
0 & 1.6956 & 0.2826 & 0.0420 \\
0 & -0.0231 & -0.0039 & -0.0006 \\
0 & 0 & 5.0000 & 0 \\
0 & 0.3500 & 0 & 0 \\
1 & 0 & 0 & 0 \\
0 & 1.0000 & 0 & 0
\end{array}\right], \mathbf{D}=\left[\begin{array}{lllll}
0 & 0 & 0 & 0 & 0.9067 \\
0 & 0 & 0 & 0 & 0.4515 \\
0 & 0 & 0 & 0 & 0 \\
0 & 0 & 0 & 0 & 0 \\
0 & 0 & 0 & 0 & 0 \\
0 & 0 & 0 & 0 & 0
\end{array}\right]
\end{aligned}
$$


We shall regard the coefficients of this system are interval entities since the nominal values in question are experimentally determined. The system is exponentially stable with spectrum

$$
\operatorname{Spec}(A)=\left[\begin{array}{ccc}
-1.4390 & + & \jmath 16.7091 \\
-1.4390 & - & \jmath 16.7091 \\
-0.4297 & + & \jmath 8.0045 \\
-0.4297 & - & \jmath 8.0045
\end{array}\right]
$$

and the maximal singular value of the system function has at $0.903 \mathrm{~dB}$ its highest peak of value 32.5166 $\mathrm{Db}$, fact which gives an estimation of the $\mathcal{H}_{\infty}$-norm of the transfer function as

$$
\left\|\mathbf{D}+\mathbf{C}(s I-\mathbf{A})^{-1} \mathbf{B}\right\|_{\mathcal{H}_{\infty}} \approx 42.2474
$$

Let $X_{\mathrm{R}}$ and $Y_{\mathrm{R}}$ be the stabilizing solutions to the bounded-real Riccati algebraic equations associated with $\Sigma$. By balancing them we obtain the following set of nominal bounded-real singular values of the system

$$
\boldsymbol{\Xi}=\{18.7990, \quad 16.1204,6.6772,6.4024\}
$$

The Matrix-Cube based robust solution has been obtained by solving (38) for $L=28$. The set of generalized bounded-real singular values are obtained by balancing the robust bounded-realness certificates which are components of the solution to (38). More precisely one obtains :

$$
\Xi=\left\{\begin{array}{llll}
0.8256, & 0.6466, & 0.3531, & 0.0437
\end{array}\right\}
$$

The estimated level of uncertainty is $\widehat{\Gamma}=11.53 \% .{ }^{1}$ As the reader can notice from the figure, the robustly reduced order system performs equally well at low and high frequencies as it does the nominal reduced order one. However, for the range of medium frequencies, the former one recovers slightly better the frequency behavior of full-order system largest singular value.

\section{CONCLUDING REMARKS AND FUTURE WORK}

A general robust dissipativity framework has been proven to represent the adequate framework for solving the model reduction problem for uncertain linear systems. Although in general is NP-hard to compute exactly, the generalized dissipative singular values - the corner stones of the balanced truncation order reduction technique we have adopted in our paper, we have shown that using the novel results from robust semi-definite optimization known as Matrix-Cube Theory, one can build tight bounds on the maximal uncertainty level for which robust dissipativity certificates exists together with these certificates. This has enabled us to generalize the dissipative order reduction theory to uncertain systems and to build a computationally tractable algorithm to develop a suboptimal solution.

It would be in our opinion extremely interesting and practically relevant to extend these results for LFT models of uncertain linear systems. The true difficulty (which is still an open problem) is that the level of conservativeness of any robust relaxation of semi-infinite systems of LMIs (including the Matrix-Cube type) is in this case difficult to be estimated.

\section{REFERENCES}

[1] L. Andersson, A. Rantzer, and C. L. Beck, Model Comparison and Simplification, Int. Journal of Nonlinear and Robust Control, Feb. (1999)

[2] B. D. O. Anderson, Controller design. Moving from theory to practice, IEEE Conference on Decision and Control, 1992.

[3] B. D. O.'Anderson and Y. Liu, Controller Reduction : Concepts and Approaches, IEEE T.A.C vol. 34, no. 8, August 1989.

[4] F.D.Barb, A. Ben-Tal, A. Nemirovski, Robust dissipativity of interval uncertain linear systems, SIAM Journal of Control and Optimization 41(6), pp: 1661 - 1695, (2003).

[5] C. L. Beck, J. Doyle, K. Glover, Model Reduction of Multi-Dimensional and Uncertain Systems, IEEE Transactions on Automatic Control, Vol. 41, Issue 10, pp. 1466-1477, Oct. (1996)

\footnotetext{
${ }^{1}$ By solving only the stabilizability part of (38) we have obtained a very good stabilizability margin which allows the corresponding $\widehat{\Gamma}$ to run up to $53.11 \%$ !
} 


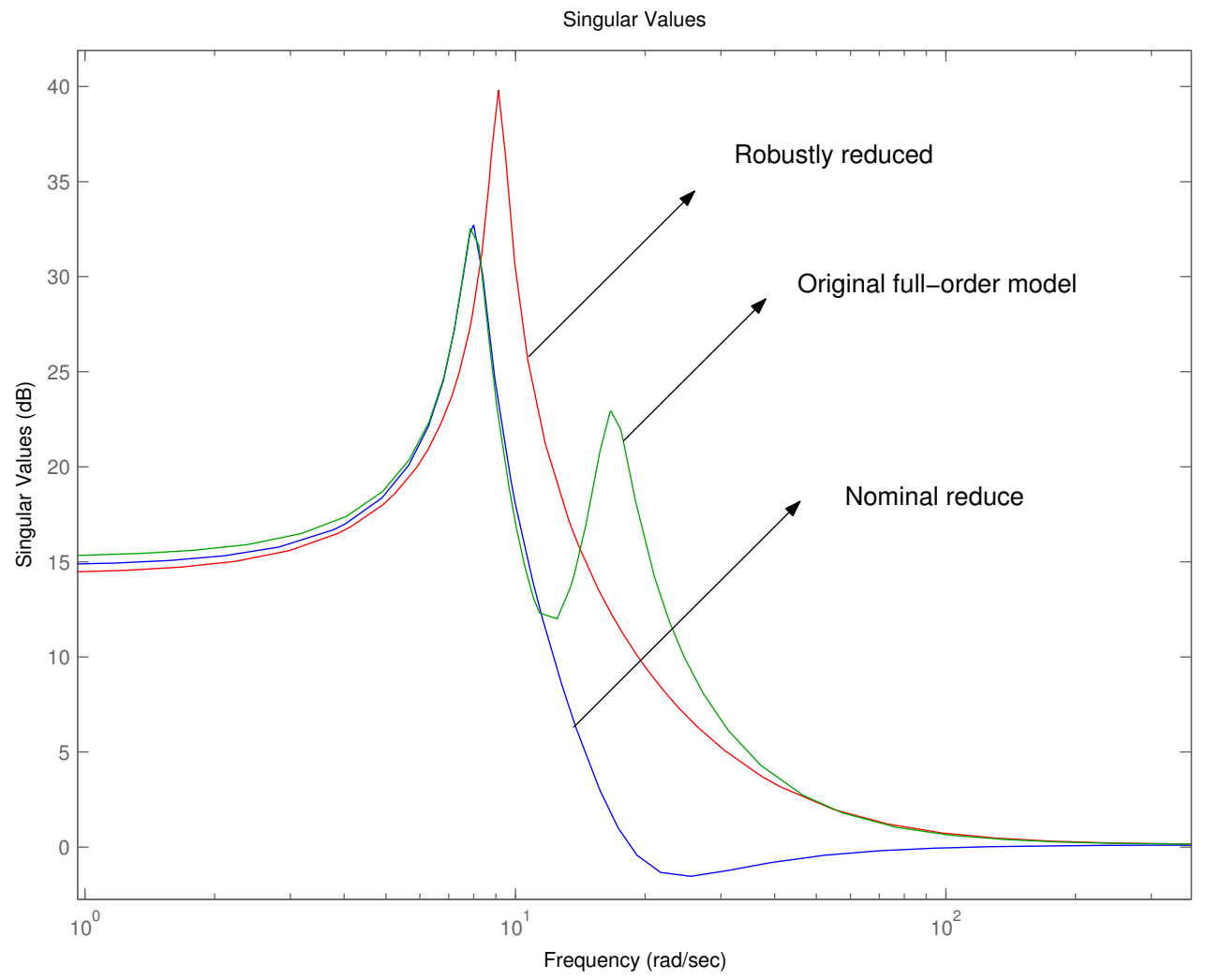

Fig. 1. The maximal singular values of the original full-sized, nominal reduced and robustly reduced systems

[6] C. L. Beck, J. Doyle, A Necessary and Sufficient Minimality Condition for Uncertain Systems, IEEE Transactions on Automatic Control, Vol. 44, Issue 10, pp. 1802-1813, Oct. (1999)

[7] C. L. Beck, P. Benedotti, Model reduction methods for unstable uncertain systems, in Proc. of the IEEE CDC, San Diego, CA (1997)

[8] C. L. Beck and R. D'Andrea, Simplification of Spatially Distributed Systems, Proceedings of the IEEE CDC, Arizona, Dec. (1999)

[9] C. L. Beck, Model reduction and minimality for uncertain systems, PhD dissertation, California Institute of Technology, 1996.

[10] C. L. Beck, On Formal Power Series Representations for Uncertain Systems, IEEE Trans. on Automatic Control, Feb, 2001.

[11] P. Bendotti and C. L. Beck, On the Role of LFT Model Reduction Methods in Robust Controller Synthesis for a Pressurized Water Reactors, IEEE Trans. on Controls Systems Technology, March 1999

[12] C. L. Beck and P. Bendotti, Model Reduction Methods for Unstable Uncertain Systems, Proceedings of the IEEE CDC, San Diego, CA, 1997.

[13] C. L. Beck, J. Doyle and K. Glover, Model reduction of multidimensional and uncertain systems, IEEE T.A.C vol. 41, 10, pp. 1466-1477, 1996.

[14] C. L. Beck and J. Doyle, A necessary and suficient minimality condition for uncertain systems, IEEE T.A.C, vol. 44, no. 10, pp. $1802-1813,1999$.

[15] A. Ben-Tal and A. Nemirovski, On tractable approximations of uncertain linear matrix inequalities affected by interval uncertainty, SIAM J. Optimization, (2002).

[16] A. Ben-Tal, A. Nemirovski, Lectures on Modern Convex Optimization, SIAM-MPS Series on Optimization, SIAM Publications, Philadelphia, 2001.

[17] A. Ben-Tal, A. Nemirovski, C. Roos, Extended Matrix-Cube theorems with applications to $\mu$-theory in control, private communication, downloadable from http://iew3.technion.ac.il/Labs/Opt/opt/Pap/MatrCube.pdf

[18] S. Boyd, L. El Ghaoui, E. Feron and V. Balakrishnan, Linear Matrix Inequalities in System and Control Theory, SIAM Studies in Applied Mathematics, vol. 15, (1994).

[19] G. Dullerud F. Paganini, A Course in Robust Control Theory. A Convex Approach, Series: Texts in Applied Mathematics, Vol. 36, Springer Verlag,

[20] L. El Ghaoui, F. Oustry and H. Lebret, Robust solutions to uncertain semi-definite programs, SIAM Journal of Optimization, vol. 9, No. 1, pp. 33-52, (1998).

[21] D. F. Enns, Model reduction for control system design, PhD dissertation, Stanford University, 1984.

[22] K. Glover, All optimal Hankel norm approximations of linear multivariable systems and their $\mathbb{L}_{\infty}$-error bounds, Int. J. Contr., vol. 39, pp. 1115-1193, 1984. 
[23] S. Gugercin, A. C. Antoulas, A survey of model reduction by balanced truncations and some new results, to appear in Int.. J. Control (2004))

[24] P. Harshavardhana, Model-reduction methods in control and signal processing, PhD Dissertation, USC-EE, 1984.

[25] V. Ionescu, C. Oară and M. Weiss, Generalized Riccati theory and robust control. A Popov function approach, John Wiley \& Sons Ltd., Great Britain, 1999.

[26] T. Iwasaki, S. Hara, Well-posedness of feedback sytsems. Insights into exact robustness analysis and approximate computations, IEEE T.A.C. 43 , pp. 619-630, (1998)

[27] E. A. Jonckheere and L. M. Silverman, A new set of invariants for linear systems - Application to reduced order compensator design, IEEE TAC vol. 28, pp. 953-964, 1983

[28] I. Emre Köse, IQC-based $\mathcal{L}_{2}$-control of linear periodic systems, Syst. \& Contr. Letters 47, pp: 199-209, (2002).

[29] R. Lind, M. Brenner, Robust Aeroservoelatic stability analysis, Advances in Industrial Control series, Springer-Verlag,(1999)

[30] D. G. Meyer, A fractional approach to model reduction, Proc. ACC, 1988.

[31] B. C. Moore, Principal component analysis in linear systems. Controllability, observability and model reduction, IEEE TAC, vol. 26, pp. 17-32, 1981.

[32] A. Nemirovski, Several NP-hard problems arising in robust stability analysis, Math. Control Signal \& Systems 6, pp. 99-105, (1993).

[33] P. C. Opdenacker, E. A. Jonckheere, A constraction mapping preserving balanced reduction scheme and its infinity norm error bounds, IEEE Trans. Circ. \& Syst., vol. 35, no. 2, pp. 184-189, 1988.

[34] J. R. Phillips, L. Daniel and L. M. Silveira, Guaranteed Passive Balancing Truncations for Model Order reduction, IEEE Tran. Computer-Aided Design of Int. Circ. and Syst., vol 22, No. 8, pp. 1 - 15, (2003)

[35] C.W. Scherer, Robust mixed-control and linear parametric varying control with full-block scalings, Chapter X, Advances in Linear Matrix Inequalities Methods in Control, SIAM Philadelphia, (2000).

[36] C.W. Scherer, LPV control and full block multipliers, Automatica 37 361-375, (2001).

[37] C.W. Scherer, P. Gahinet, M Chilali, Multi-objective output-feedback control via LMI optimization IEEE Trans. Auto. Contr. 42 pp.896-911, (1997).

[38] C. Scherer and S. Weiland, Linear Matrix Inequalities in Control, Dutch Institute of Systems and Control graduate course lecture notes, version 2.0, http://www.ocp. tudelft.nl/sr/personal/Scherer/lmi.pdf (April 1999).

[39] G. Scorletti, Approche Unifiée de l'Analyse et de la Commande des Systèmes par Optimisation LMI PhD Thesis Universitè de Paris XI (Orsay) France, (1997).

[40] W. Wang, J. Doyle, C. L. Beck and K. Glover, Model reduction of LFT systems, Proceedings of the $30^{\text {th }}$ IEEE Conference on Decision and Control, pp.1233 -1238, 1991.

[41] J. C. Willems, Dissipative dynamical systems, part I: General theory, Arch. Rational Mech. Anal. 45, pp. 321-351, (1971).

[42] J. C. Willems, Dissipative dynamical systems, part II: Linear systems with quadratic supply rates, Arch. Rational Mech. Anal. 45, pp. 352-393, (1971).

[43] J. C. Willems, Least squares stationary optimal control and the algebraic Riccati equation, IEEE T.A.C. 16, pp. 621-634, (1971).

[44] K. Zhou, Robust and Optimal Control, Prentice-Hall, Upper Saddle River, NJ, (1996). 